\title{
Stabilizing Control Design for a Class of Discontinuous Systems
}

\author{
Takashi NAKAKUKI*, Katsutoshi TAMURA* and Tielong Shen*
}

\begin{abstract}
This paper presents a stabilizing controller design for an affine input system with discontinuity. We pursue the uniqueness of the Filippov solution of the closed loop system in addition to the asymptotic stability. First, a typical $L_{g} V$ structure-based switching controller is considered. However, it is shown that the uniqueness of the solution of the closed loop system is not necessarily assured by the controller in the whole state space. Then, an asymptotic stabilizing controller which entirely assures the uniqueness in the whole state space is developed by modifying the $L_{g} V$ structure-based switching controller.
\end{abstract}

Key Words: differential inclusion, discontinuous system, existence and uniqueness of solution, Filippov's solution, stabilizing control

\section{Introduction}

Control and stability analysis of discontinuous systems have attracted considerable research interests in both mathematical issues and practical applications. In the mathematical issues, the definition of the solutions of differential equations with discontinuous right hand side has been proposed, and the existence and uniqueness of the solution have been also explored ${ }^{2), 4)}$. On the other hand, in practical applications, control problems of nonlinear systems with discontinuity such as stick-slip motion, backlash, switching control, etc. have been considered in the Filippov's framework ${ }^{-1)}$, 7) 10)

The questions of the existence and uniqueness of the solution of the closed loop system with discontinuous controllers were pursued by Polycarpou ${ }^{9)}$ and $\mathrm{Kim}^{6)}$. Polycarpou $^{9)}$ showed a sufficient condition for the existence and uniqueness of the Filippov solution of adaptive control systems including switching adaptive schemes. Recently, Kim ${ }^{6)}$ presented a sufficient condition for the existence of the Carathéodory solution in nonlinear systems with discontinuous switching controllers. On the other hand, controller design in practical applications has been developed ${ }^{1), 7), 8), 10)}$. Paden ${ }^{8)}$ developed a variable structure controller design of robot manipulators by rigorous nonsmooth analysis. Alvarez ${ }^{1)}$ proposed a switching control algorithm for a Coulomb friction oscillator. However, these papers did not discuss the uniqueness of the Filippov solution of the closed loop systems. Recently,

\footnotetext{
* Department of Mechanical Engineering, Graduate Division of Science and Technology, Sophia University, 7-1, Kioicho, Chiyoda-ku, Tokyo

(Received August 4, 2004)

(Revised March 15, 2004)
}

Orlov $^{7)}$ developed a chattering controller for an electrical servo-motors with discontinuity. In his paper, although the property of the solution on the discontinuous surface by the controller was examined, the uniqueness of the solution on the other discontinuity was not discussed. Sekhavat ${ }^{10)}$ showed not only a controller design for a hydraulic actuator with friction but also the existence and uniqueness of the solution of the closed loop system.

Hence, in this paper, we propose a stabilizing controller which assures the uniqueness of the Filippov solution of the closed loop system in addition to the asymptotic stability for an affine input system with discontinuity. First, we provide a sufficient condition for which any Lipschitz continuous controller can not achieve the asymptotic stability of the system. This is another sufficient condition unlike the result by Alvarez ${ }^{1)}$. Then, a switching control law, which belongs to so-called $L_{g} V$ structure-based switching control, is examined. However, it is shown that the typical discontinuous controller does not necessarily assure the uniqueness of the solution of the closed loop system in the whole state space, which gives us a motivation of the modification of the controller in order to entirely assure the uniqueness in the state space. As a main result, we provide a modified $L_{g} V$ structure-based switching controller which follows the uniqueness and asymptotic stability in the whole state space. We also extend our result to the multi-input system.

\section{Problem Statement}

Consider the plant with nonlinear discontinuity $\mu(x)$

$$
\dot{x}=A x+b(\mu(x)+u)
$$

where $x \in \mathbb{R}^{n}$ is the state vector and $u \in \mathbb{R}$ is the control input. The pair $(A, b)$ is assumed to be controllable. The 


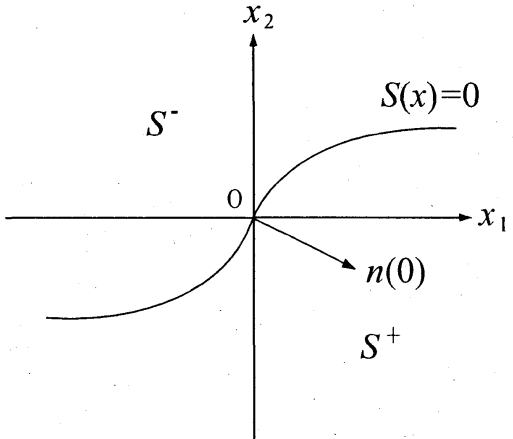

Fig. $1 \quad S(x), S^{-}$and $S^{+}$

discontinuity $\mu(x)$ is defined by

$$
\mu(x)=\left\{\begin{aligned}
m_{1} & \text { if } S(x)>0 \\
-m_{2} & \text { if } S(x)<0
\end{aligned}\right.
$$

where $m_{1}, m_{2}$ are positive constants. $S(x)$ is a smooth surface with $S(0)=0$ which divides the state space into the domains $S^{+}$and $S^{-}$as shown in Fig. 1. Without loss of generality we assume that $A$ is an asymptotic stable matrix. The purpose of the controller design is to find a control law $u(x)$ which assures the asymptotic stability of the origin and the uniqueness of the solution of the closed loop system. Define $f(x)$ by

$$
f(x)=A x+b(\mu(x)+u(x))
$$

As shown in Proposition 3.2 stabilizing control law $u(x)$ should be a discontinuous function. Thus, the closed loop system should be described by the differential inclusion form

$$
\dot{x} \in K[f](x)
$$

where $K[\cdot]$ is the Filippov set ${ }^{4)}$ (See Appendix for definition). For the origin $x=0$ being an equilibrium point, the following is required.

$$
0 \in K[f](0)
$$

Since system (4) may have multiple equilibria as shown in Example 3.1, we must show that $x=0$ is only one stable equilibrium point.

\section{Controller Design and Stability Analysis}

First, we discuss the stability property of the free system of (1)

$$
\dot{x}=\bar{f}(x)=A x+b \mu(x)
$$

Since $S(0)=0$,

$$
K[\bar{f}](0)=\left\{\zeta \in \mathbb{R} \mid \zeta=\left(\alpha m_{1}-(1-\alpha) m_{2}\right) b, 0 \leq \alpha \leq 1\right\}
$$

Thus, $0 \in K[\bar{f}](0)$, and $x=0$ is a candidate of the equilibrium point. Let $n(x)$ be the normal vector to the surface $S(x)$ at a point $x$ directed from $S^{-}$to $S^{+}$as shown in Fig. 1. Let $x$ be a point on the surface $S(x)$, and $\bar{f}^{-}(x)$ and $\bar{f}^{+}(x)$ be limiting values where $x$ is approached from $S^{-}$and $S^{+}$respectively. The projection of $\bar{f}^{-}$and $\bar{f}^{+}$to $n(x)$ is defined as

$$
\begin{aligned}
& \bar{f}_{n}^{+}(x)=\left\langle n(x), \bar{f}^{+}(x)\right\rangle \\
& \bar{f}_{n}^{-}(x)=\left\langle n(x), \bar{f}^{-}(x)\right\rangle
\end{aligned}
$$

Proposition 3.1. If $\vec{f}_{n}^{+}(0)>0$ and $\vec{f}_{n}^{-}(0)<0$, the origin $x=0$ of system (6) is not stable. We call this $d$-unstable in this paper which comes from unstability induced by discontinuity.

Proof. Consider the trajectory starting from $x(0)=$ $\delta_{1} b \in B_{\delta}(0) \cap S^{+}$for sufficiently small $\delta_{1}>0$ where $B_{\delta}(0)$ is a $\delta$-neighbourhood of the origin. Then, the trajectory is dominated by

$$
\dot{x}=A x+b m_{1}
$$

which yields the following solution

$$
x(t)=e^{A t} \delta_{1} b+\left(e^{A t}-I\right) A^{-1} b m_{1}
$$

Since $A$ is stable from the assumption and $\delta_{1}$ is a sufficiently small number,

$$
x(t) \simeq\left(e^{A t}-I\right) A^{-1} b m_{1}
$$

For small $t_{1}>0$,

$$
e^{A t} \simeq I+A t, \quad \forall t \in\left[0, t_{1}\right]
$$

Moreover, since $\bar{f}_{n}^{+}(0)>0, x(t) \in S^{+}$for all $t \in\left[0, t_{2}\right]$ where $t_{2}$ is a small number. Thus, we have

$$
x(t) \simeq m_{1} b t, \quad \forall t \in\left[0, t^{+}\right]
$$

where $t^{+}=\min \left(t_{1}, t_{2}\right)$. We can also derive the similar statement for the trajectory starting from $x(0)=\delta_{1} b \in$ $B_{\delta}(0) \cap S^{-}$. Hence, from the definition of stable (e.g. Khalil ${ }^{5)}$, pp.112, Definition 4.1), we can conclude that the system is not stable and any trajectory can not approach to the origin.

When a control law $u(x)$ for system (1) is Lipschitz continuous, the same discussion as described in Proposition 3.1 is applied to obtain the following.

Proposition 3.2. Let the origin of system (6) be d-unstable. Then any Lipschitz continuous controller $u(x)$ with $u(0)=0$ can not achieve the asymptotic stability of the origin of system (1).

Proof. Since the controller $u(x)$ is Lipschitz, it can be approximated by

$$
u(x)=k x, \quad k \in \mathbb{R}^{1 \times n},\|k\|<+\infty, \quad x \in D
$$


where the domain $D$ is a neighbourhood of the origin. As a result, the closed loop system is given by

$$
\dot{x}=A x+b(k x+\mu(x))=(A+b k) x+b \mu(x), \quad \forall x \in D
$$

The left of the proof can be done with the similar manner in Proposition 3.1.

Remark 3.1. It is stated in the literature ${ }^{1)}$ that a Coulomb friction oscillator, which is modeled with $A \in$ $\mathbb{R}^{2 \times 2}, b=\left[\begin{array}{ll}0 & 1\end{array}\right]^{T} \in \mathbb{R}^{2}$ in (1) and $S(x)=-x_{2}$ in (2), can not be asymptotically stabilized via any continuous control algorithm because the closed loop system possesses a non-trivial set of equilibrium points. The conclusion is derived from the conditions $\bar{f}_{n}^{+}(0)<0$ and $\bar{f}_{n}^{-}(0)>0$ on $x_{2}=0$ around the origin for the second order specific model.

Remark 3.2. Consider discontinuous system (6) Contrary to d-unstable, the case satisfying the condition

$$
\bar{f}_{n}^{+}(0)<0, \quad \bar{f}_{n}^{-}(0)>0
$$

does not necessarily imply the stability of the origin of system (6). See the following example.

Example 3.1. Consider the following system

$$
\dot{x}=\left[\begin{array}{cc}
0 & 1 \\
-2 & -3
\end{array}\right] x+\left[\begin{array}{c}
0 \\
\mu(x)
\end{array}\right]
$$

The discontinuity is given by

$$
\mu(x)=\left\{\begin{aligned}
1 & \text { if } S(x)=x_{1}-x_{2}>0 \\
-1 . & \text { if } S(x)=x_{1}-x_{2}<0
\end{aligned}\right.
$$

Then we have

$$
n(0)=\left[\begin{array}{c}
1 \\
-1
\end{array}\right], \quad \bar{f}^{+}(0)=\left[\begin{array}{l}
0 \\
1
\end{array}\right], \quad \bar{f}^{-}(0)=\left[\begin{array}{c}
0 \\
-1
\end{array}\right]
$$

$$
\bar{f}_{n}^{+}(0)=-1, \bar{f}_{n}^{-}(0)=1
$$

The trajectories around the origin are given in Fig. 2. Note that system (17) has multiple equilibria

$$
x_{\mathrm{e} 0}=\left[\begin{array}{l}
0 \\
0
\end{array}\right], \quad x_{\mathrm{e} 1}=\left[\begin{array}{c}
0.5 \\
0
\end{array}\right], \quad x_{\mathrm{e} 2}=\left[\begin{array}{c}
-0.5 \\
0
\end{array}\right]
$$

$x_{\mathrm{e} 1}$ and $x_{\mathrm{e} 2}$ are locally stable equilibria.

Now, we consider the stabilizing problem of system (1). Since $A$ is a stable matrix there exists a symmetric positive definite matrix $P$ for any given $Q=Q^{T}>0$ which satisfies the following Lyapunov equation

$$
P A+A^{T} P=-Q
$$

Take $u(x)$ as

$$
u(x)=-\gamma \operatorname{sgn}\left(b^{T} P x\right)
$$

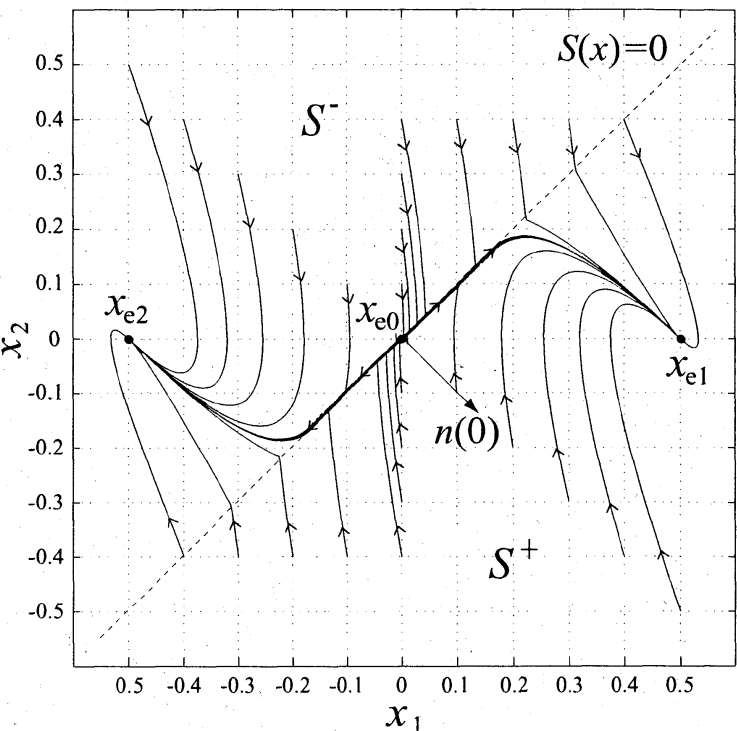

Fig. 2 Trajectories of system (17)

where $\gamma>\max \left(m_{1}, m_{2}\right)=m$. Then, the closed loop system is given by

$$
\dot{x}=A x+b\left(\mu(x)-\gamma \operatorname{sgn}\left(b^{T} P x\right)\right)
$$

Let $V(x)$ be a Lyapunov function candidate given by

$$
V(x)=\frac{1}{2} x^{T} P x
$$

Since the right hand side of system (24) is discontinuous, by calculating $\dot{\tilde{V}}(x)$ (See Appendix for definition) along the trajectories of system (24), we obtain

$$
\begin{aligned}
\dot{\tilde{V}}(x)= & \left\{v \in \mathbb{R} \mid v=-\frac{1}{2} x^{T} Q x+x^{T} P b K[\mu(x)]\right. \\
& \left.+x^{T} P b K\left[-\gamma \operatorname{sgn}\left(b^{T} P x\right)\right]\right\}
\end{aligned}
$$

Since $\max _{\zeta \in K[\mu](x)}|\zeta|=m$,

$v \leq-\frac{1}{2} x^{T} Q x-(\gamma-m)\left|x^{T} P b\right|<0, \quad \forall x \neq 0, \forall v \in \dot{\tilde{V}}(x)$

Notice that controller (23) is a so-called $L_{g} V$ structurebased switching controller. The Lyapunov stability theory ${ }^{11)}$ for nonsmooth systems is given under the condition of the existence and uniqueness of the Filippov solution of the closed loop system. As a result, we should also examine the existence and uniqueness of the Filippov solution of the closed loop system (24). The following example show that the uniqueness of the Filippov solution of the closed loop system is not assured in the whole state space while the set valued derivative $\dot{\tilde{V}}(x)$ is negative definite.

Example 3.2. Consider the following system

$$
\dot{x}=\left[\begin{array}{cc}
0 & 1 \\
-2 & -3
\end{array}\right] x+\left[\begin{array}{c}
0 \\
\mu(x)+u(x)
\end{array}\right]
$$


Unlike Example 3.1 the discontinuity is given by

$$
\mu(x)=\left\{\begin{aligned}
1 & \text { if } S(x)=10 x_{1}+x_{2}>0 \\
-1 & \text { if } S(x)=10 x_{1}+x_{2}<0
\end{aligned}\right.
$$

From $(23), u(x)$ is given by

$$
u=-\gamma \operatorname{sgn}\left(b^{T} P x\right)=-2 \operatorname{sgn}\left(0.25 x_{1}+0.25 x_{2}\right)
$$

where $P$ is given by

$$
P=\left[\begin{array}{ll}
1.25 & 0.25 \\
0.25 & 0.25
\end{array}\right]
$$

from (22) for given $Q=I$ (unit matrix). Also let $V(x)$ be a Lyapunov function candidate given by (25). Then, we can conclude that $\dot{\tilde{V}}(x)<0$ for all $x \neq 0$ from $(27)$. We can also show that there exists a point failing to satisfy the sufficient condition for the uniqueness of the solution by Filippov ${ }^{4)}$ (See Theorem A.1). In fact, define $\Omega=\left\{x \in \mathbb{R}^{2} \mid S(x)=0\right\}$. Consider the point $x^{*} \in\left\{x \in \Omega \mid b^{T} P x>0\right\}$. Then, we have

$$
\begin{aligned}
& f^{+}\left(x^{*}\right)=\left[\begin{array}{c}
x_{2} \\
-2 x_{1}-3 x_{2}-1
\end{array}\right] \\
& f^{-}\left(x^{*}\right)=\left[\begin{array}{c}
x_{2} \\
-2 x_{1}-3 x_{2}-3
\end{array}\right]
\end{aligned}
$$

By calculating $f_{n}^{+}\left(x^{*}\right)$ and $f_{n}^{-}\left(x^{*}\right)$ with $n\left(x^{*}\right)=[10,1]^{T}$,

$$
\begin{aligned}
& f_{n}^{+}\left(x^{*}\right)=-2 x_{1}+7 x_{2}-1=-72 x_{1}-1 \\
& f_{n}^{-}\left(x^{*}\right)=-2 x_{1}+7 x_{2}-3=-72 x_{1}-3
\end{aligned}
$$

Hence, on the set

$$
\bar{N}=\left\{x \in \mathbb{R}^{2} \mid S(x)=0,-3 / 72<x_{1}<-1 / 72\right\}
$$

we have

$$
f_{n}^{+}\left(x^{*}\right)>0, \quad f_{n}^{-}\left(x^{*}\right)<0
$$

Then, the two solutions starting from $\bar{N}$ are given by

$$
\begin{aligned}
& x(t)=e^{A t} x(0)-\int_{0}^{t} e^{A(t-\tau)} b d \tau \\
& x(t)=e^{A t} x(0)-3 \int_{0}^{t} e^{A(t-\tau)} b d \tau
\end{aligned}
$$

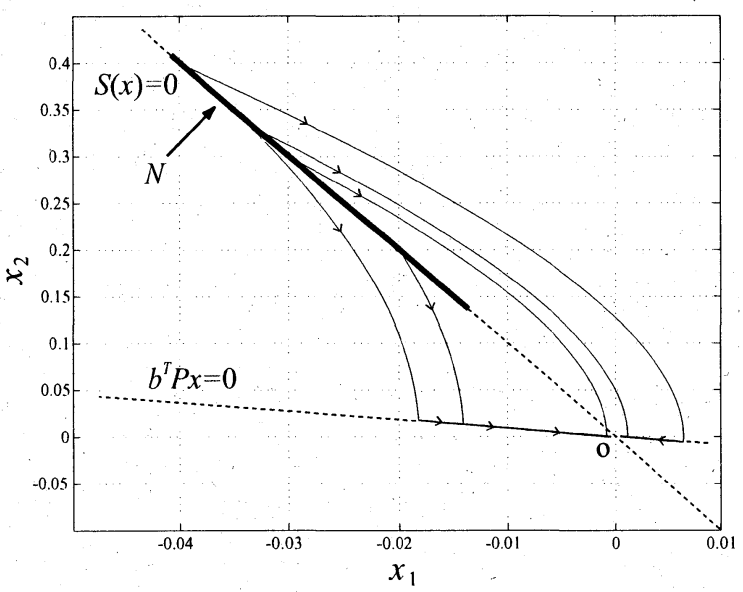

Fig. 3 Trajectories of system (28)

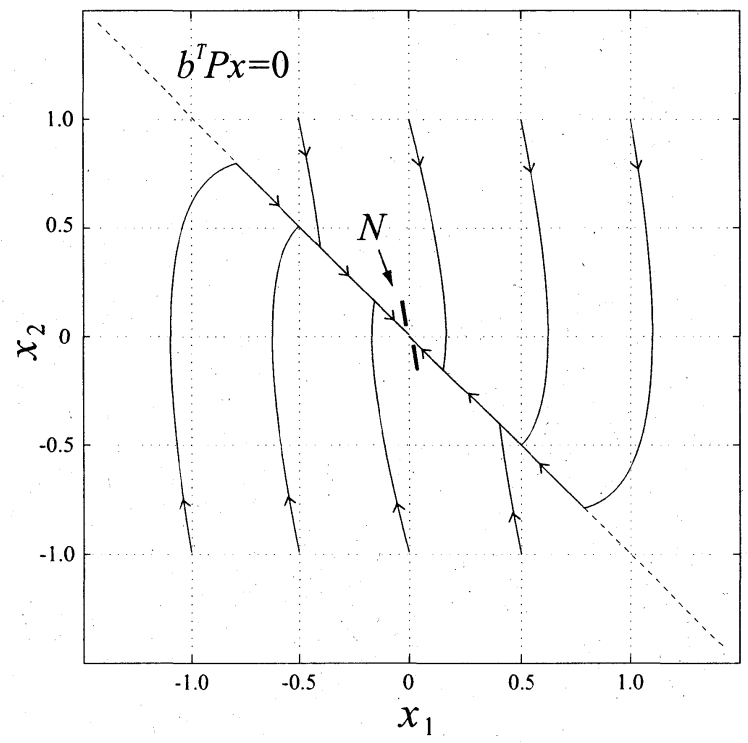

Fig. 4 Trajectories of system (28)

where

$$
A=\left[\begin{array}{cc}
0 & 1 \\
-2 & -3
\end{array}\right], b=\left[\begin{array}{l}
0 \\
1
\end{array}\right]
$$

In a similar way, by considering the point $x^{*} \in\{x \in$ $\left.\Omega \mid b^{T} P x<0\right\}$, we obtain the following set

$$
N=\left\{x \in \mathbb{R}^{2}|S(x)=0,1 / 72<| x_{1} \mid<3 / 72\right\}
$$

such that (35) is satisfied, which implies that the condition for the uniqueness of the Filippov solution of the closed loop system is not satisfied. As shown in Fig. 3 trajectories starting from $x(0) \in N$ have two different behaviors. Fig. 4 shows simulation results starting from $x(0) \notin N$. Fortunately, all trajectories converge to the origin due to (27).

Theorem 3.1. Consider system (1). Let the control law be given by (23). Assume that $x(0) \notin \Omega_{o}=\{x \in$ $\left.\mathbb{R}^{n} \mid S(x)=0, x \neq 0\right\}$. Then, the Filippov solution $x(t)$ of system (24) converges to the origin as $t \rightarrow \infty$ and the uniqueness of the solution is assured.

Proof. From Lyapunov stability theory ${ }^{11)}$ and the negative definiteness of the set valued derivative $\dot{\tilde{V}}(x)$, the convergence of trajectories to 0 has already been confirmed. Hence, we examine the existence and uniqueness of the Filippov solution of (24).

[existence] On an arbitrary compact set $D \subset \mathbb{R}^{n}$ the right hand side of (24) satisfies

$$
\begin{aligned}
& \left\|A x+b\left(\mu(x)-\gamma \operatorname{sgn}\left(b^{T} P x\right)\right)\right\| \\
& \leq\|A x\|+\|b\|\left(\max \left(m_{1}, m_{2}\right)+\gamma\right) \text { a.e. } \\
& \leq L
\end{aligned}
$$

where $L$ is a constant. Thus the right hand side of (24) 
satisfies Condition B (See Appendix) which is a sufficient condition for the existence of the Filippov solution.

[uniqueness] System (24) has two discontinuous surfaces by $\mu(x)$ and $\operatorname{sgn}\left(b^{T} P x\right)$. Define $\Omega_{o}$ and $\Omega_{c}$ by

$$
\begin{aligned}
& \Omega_{o}=\left\{x \in \mathbb{R}^{n} \mid S(x)=0, x \neq 0\right\} \\
& \Omega_{c}=\left\{x \in \mathbb{R}^{n} \mid b^{T} P x=0, x \neq 0\right\}
\end{aligned}
$$

Then it is clear that in $\mathbb{R}^{n}-\left(\Omega_{o} \cup \Omega_{c}\right)$ the vector field $f(x)$ satisfies Lipschitz condition where

$$
f(x)=A x+b\left(\mu(x)-\gamma \operatorname{sgn}\left(b^{T} P x\right)\right)
$$

Thus we focus on the uniqueness of the solution on $\Omega_{o} \cup \Omega_{c}$. Let $x$ be a point on $\Omega_{c}$. Then we have

$$
\begin{aligned}
& f^{+}(x)=A x+b(\mu(x)-\gamma) \\
& f^{-}(x)=A x+b(\mu(x)+\gamma)
\end{aligned}
$$

where $f^{-}(x)$ and $f^{+}(x)$ be limiting values where $x$ is approached from $\Omega_{c}^{-}=\left\{x \in \mathbb{R}^{n} \mid b^{T} P x<0\right\}$ and $\Omega_{c}^{+}=\left\{x \in \mathbb{R}^{n} \mid b^{T} P x>0\right\}$ respectively. Let $n_{c}(x)$ be the normal vector to the surface $\Omega_{c}$ at a point $x$ directed from $\Omega_{c}^{-}$to $\Omega_{c}^{+}$. The projection of $f^{-}$and $f^{+}$to $n_{c}(x)$ is defined as

$$
\begin{aligned}
& f_{n_{c}}^{+}(x)=\left\langle n_{c}(x), f^{+}(x)\right\rangle \\
& f_{n_{c}}^{-}(x)=\left\langle n_{c}(x), f^{-}(x)\right\rangle
\end{aligned}
$$

Since $\gamma>\max \left(m_{1}, m_{2}\right)$, we obtain

$$
f_{n}^{+}(x)-f_{n}^{-}(x)=b^{T} P b(-2 \gamma)<0 \text { if } x \in \Omega_{c} \text { and } x \notin \Omega_{o}
$$

which implies that the uniqueness of the solution on $\Omega_{c}$ is assured according to Theorem A.1. Next let $x$ be a point on $\Omega_{o}$. Then, in the region $\Omega_{c}^{+}$we have

$$
\begin{aligned}
& f_{n}^{+}(x)=n^{T} A x-\gamma n^{T} b+m_{1} n^{T} b \\
& f_{n}^{-}(x)=n^{T} A x-\gamma n^{T} b-m_{2} n^{T} b
\end{aligned}
$$

In a similar way, in the region $\Omega_{c}^{-}$we also obtain

$$
\begin{aligned}
& f_{n}^{+}(x)=n^{T} A x+\gamma n^{T} b+m_{1} n^{T} b \\
& f_{n}^{-}(x)=n^{T} A x+\gamma n^{T} b-m_{2} n^{T} b
\end{aligned}
$$

However, as shown in Example 3.2, there exist some points such that the inequality $f_{n}^{+}(x)-f_{n}^{-}(x)<0$ with respect to $(46)$ or $(47)$ is not satisfied where the sufficient condition for the uniqueness is not satisfied. Hence, avoiding $x(0) \in \Omega_{o}$, the uniqueness of the solution of $(24)$ is assured.

Furthermore, we pursue the uniqueness of the solution in the whole state space unlike Theorem 3.1. Example 3.2 and Theorem 3.1 give us an insight, that is, the controller should modify the vector field on $\Omega_{o}$.
Theorem 3.2. Consider system (1). Let the control law be given by

$$
u=-\gamma_{1} \operatorname{sgn}\left(b^{T} P x\right)-\gamma_{2} \operatorname{sgn}\left(\frac{\partial S}{\partial x} b\right) \operatorname{sgn}(S(x))
$$

where $\gamma_{1}>\max \left(m_{1}, m_{2}\right)+\gamma_{2}$ and $\gamma_{2}>\left(m_{1}+m_{2}\right) / 2$. Suppose that $(\partial S / \partial x) b \neq 0$ for all $x \in \mathbb{R}^{n}$. Then, the origin $x=0$ is globally asymptotically stable and the uniqueness of the Filippov solution of the closed loop system is assured in the whole state space.

Proof. The closed loop system is given by

$\dot{x}=A x+b\left\{\mu(x)-\gamma_{1} \operatorname{sgn}\left(b^{T} P x\right)-\gamma_{2} \operatorname{sgn}\left(\frac{\partial S}{\partial x} b\right) \operatorname{sgn}(S(x))\right\}$

To replace $\mu(x)$ by $\bar{\mu}(x)=\mu(x)-\gamma_{2} \operatorname{sgn}\left(\frac{\partial S}{\partial x} b\right) \operatorname{sgn}(S(x))$, the similar derivation from (23) to (27) can be applied to obtain the negative definiteness of the set valued derivative $\dot{\tilde{V}}(x)$ of a Lyapunov function candidate $V(x)$ given by (25) because of $\gamma_{1}>\max _{\zeta \in K[\bar{\mu}(x)]}|\zeta|=\max \left(m_{1}, m_{2}\right)+\gamma_{2}$. The existence of the Filippov solution of (49) is also shown in a similar way to the proof of Theorem 3.1. Thus we here focus on the uniqueness of the Filippov solution of (49). Let $x$ be a point on $\Omega_{0}$. Then we have

$$
\begin{aligned}
& f^{+}(x)=A x+b\left\{m_{1}-\gamma_{1} \operatorname{sgn}\left(b^{T} P x\right)-\gamma_{2} \operatorname{sgn}\left(\frac{\partial S}{\partial x} b\right)\right\} \\
& f^{-}(x)=A x+b\left\{-m_{2}-\gamma_{1} \operatorname{sgn}\left(b^{T} P x\right)+\gamma_{2} \operatorname{sgn}\left(\frac{\partial S}{\partial x} b\right)\right\}
\end{aligned}
$$

Since $\gamma_{2}>\left(m_{1}+m_{2}\right) / 2$, we obtain

$$
\begin{aligned}
f_{n}^{+}(x)-f_{n}^{-}(x) & =\frac{\partial S}{\partial x} b\left\{m_{1}+m_{2}-2 \gamma_{2} \operatorname{sgn}\left(\frac{\partial S}{\partial x} b\right)\right\} \\
& <0, \quad \forall x \in \Omega_{o}
\end{aligned}
$$

which implies that the uniqueness of the solution on $\Omega_{o}$ is assured according to Theorem A.1. The uniqueness of the solution on $\Omega_{c}$ can be shown in a similar way to the proof of Theorem 3.1. Since for all Filippov solutions starting from any initial conditions the existence and uniqueness of the solution are established, from Theorem A.2 with $D=\mathbb{R}^{n}$ and radially unboundedness of $(25)$ we can conclude that $x=0$ is globally asymptotically stable.

Example 3.3. Consider system (28) again. In order to assure the uniqueness in addition to the asymptotic stability, we employ the following controller

$$
u=-4 \operatorname{sgn}\left(0.25 x_{1}+0.25 x_{2}\right)-2 \operatorname{sgn}\left(10 x_{1}+x_{2}\right)
$$

according to (48) where $(\partial S / \partial x) b=1$ for all $x$. Fig. 5 shows some simulation results for the closed loop system. In this case, the origin $x=0$ is globally asymptotically stable and the uniqueness is assured in the whole state space unlike Fig. 4. 


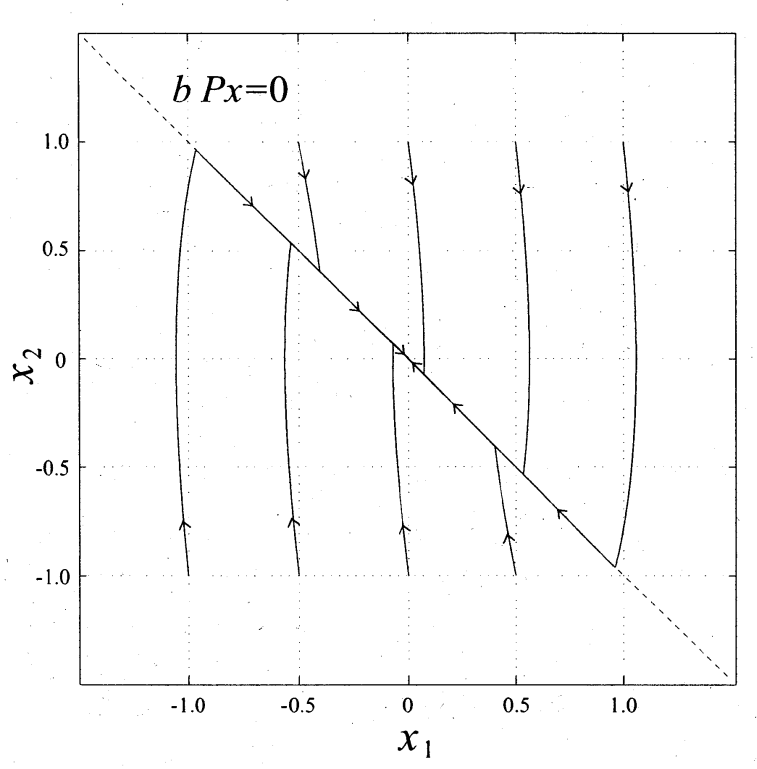

Fig. 5 Trajectories of system (28)

\section{Multi-input Case}

In this section we will show a slight generalization of Theorem 3.2 to enlarge single input system (1) to a multiinput system.

Consider the following multi inputs system.

$$
\dot{x}=A x+B(\mu(x)+u)
$$

where $x \in \mathbb{R}^{n}$ is the state vector and $u \in \mathbb{R}^{m}$ is the control input. The pair $(A, B)$ is assume to be controllable. $\mu(x) \in \mathbb{R}^{m}$ is given by

$$
\mu_{i}(x)=\left\{\begin{aligned}
m_{1}^{i} & \text { if } S_{i}(x)>0 \\
-m_{2}^{i} & \text { if } S_{i}(x)<0
\end{aligned}\right.
$$

where $\mu_{i}(x)$ is the $i$ th component of $\mu(x)$, and $m_{1}^{i}, m_{2}^{i}$ are positive constants for each $i=1,2, \ldots, m$. For each $i=1,2, \ldots, m, S_{i}(x)$ is a smooth surface with $S_{i}(0)=0$ which divides the state space into the domains $S_{i}^{+}$and $S_{i}^{-}$. Without loss of generality we assume that $A$ is an asymptotic stable matrix.

Theorem 4.1. Consider system (53). Let the control law be given by

$$
u=\left[\begin{array}{c}
-\gamma_{1} \operatorname{sgn}\left(b_{1}^{T} P x\right)-\bar{\gamma}_{1} \operatorname{sgn}\left(\frac{\partial S_{1}}{\partial x} b_{1}\right) \operatorname{sgn}\left(S_{1}(x)\right) \\
-\gamma_{2} \operatorname{sgn}\left(b_{2}^{T} P x\right)-\bar{\gamma}_{2} \operatorname{sgn}\left(\frac{\partial S_{2}}{\partial x} b_{2}\right) \operatorname{sgn}\left(S_{2}(x)\right) \\
\vdots \\
-\gamma_{m} \operatorname{sgn}\left(b_{m}^{T} P x\right)-\bar{\gamma}_{m} \operatorname{sgn}\left(\frac{\partial S_{m}}{\partial x} b_{m}\right) \operatorname{sgn}\left(S_{m}(x)\right)
\end{array}\right]
$$

where $\gamma_{i}>\max \left(m_{1}^{i}, m_{2}^{i}\right)+\bar{\gamma}_{i}$ and $\bar{\gamma}_{i}>\left(m_{1}^{i}+m_{2}^{i}\right) / 2$ for each $i=1,2, \ldots, m$,

$$
\left[\begin{array}{c}
b_{1}^{T} P x \\
b_{2}^{T} P x \\
\vdots \\
b_{m}^{T} P x
\end{array}\right]=\left[\begin{array}{c}
b_{1}^{T} \\
b_{2}^{T} \\
\vdots \\
b_{m}^{T}
\end{array}\right] P x=B^{T} P x
$$

and $P$ is a symmetric positive definite matrix satisfying Lyapunov equation (22) for given $Q=Q^{T}>0$. Suppose that, for each $i=1,2, \ldots, m,\left(\partial S_{i} / \partial x\right) b_{i} \neq 0$ for all $x \in \mathbb{R}^{n}$. Then, the origin $x=0$ is globally asymptotically stable and the uniqueness of the solution of the closed loop system is assured in the whole state space.

\section{Conclusions}

For the purpose of the uniqueness of the solution of the closed loop system and the global asymptotic stability, an analysis for the uniqueness of the Filippov solution and controller designs by employing nonsmooth analysis have been presented for an affine system with discontinuity. A condition for which any Lipschitz continuous controller can not achieve the asymptotic stability of the system is provided. Then, a $L_{g} V$ structure-based switching controller is considered, and the uniqueness of the solution of the closed loop system is evaluated. It is shown that the typical $L_{g} V$ controller does not necessarily assure the uniqueness of the solution in the whole state space. In order to entirely establish the uniqueness in addition to the global asymptotic stability, a modification of the $L_{g} V$ controller is proposed. As a slight generalization, the result is extended to multi-input version. Our results can be extended to general nonlinear systems by replacing $b^{T} P x$ by $L_{g} V_{0}$ where $V_{0}$ is a Lyapunov function for the nominal systems.

\section{Acknowledgements}

This research is supported by the fund of Open Research Center Project from MEXT of Japanese Government (2002-2006).

\section{References}

1) J. Alvarez, I. Orlov and L. Acho: An Invariance Principle for Discontinuous Dynamic Systems with Application to a Coulomb Friction Oscillator, Journal of Dynamic Systems, Measurement, and Control, 122, 687/690 (2000)

2) J. Aubin: Differential Inclusions: Set-valued Maps and Viability Theory, Springer-Verlag, New-York (1984)

3) R. Clarke: Optimization and Nonsmooth Analysis, Society for Industrial and Applied Mathematics, Pennsylvania (1990)

4) A. Filippov: Differential equations with discontinuous right-hand side, American Mathematical Society Translations, 42, 199/231 (1964)

5) H. Khalil: Nonlinear Systems(3rd), Prentice Hall, New Jersey (2002) 
6) S-J. Kim and I-J. Ha: Existence of Carathéodory solutions in nonlinear systems with discontinuous switching feedback controllers, IEEE Transactions on Automatic Control, 49, 1167/1171 (2004)

7) Y. Orlov, L. Aguilar and J. Cadiou: Switched chattering control vs. backlash/friction phenomena in electrical servomotors, International Journal of Control, 76, 959/967 (2003)

8) B. Paden and S. Sastry: A Calculus for Computing Filippov's Differential Inclusion with Application to the Variable Structure Control of Robot Manipulators, IEEE Transactions on Circuits and Systems, 34, 73/82 (1987)

9) M. Polycarpou and P. Ioannou: On the existence and uniqueness of solutions in adaptive control systems, IEEE Transactions on Automatic Control, 38, 474/479 (1993)

10) P. Sekhavat, Q. Wu and N. Sepehri: Lyapunov-based friction compensation for accurate positioning of a hydraulic. actuator, Proceeding of the 2004 American Control Conference, Boston, 418/423 (2004)

11) D. Shevitz and B. Paden: Lyapunov stability theory of nonsmooth systems, IEEE Transactions on Automatic Control, 39, 1910/1914 (1994)

\section{Appendix A. Mathematical Preliminary}

Consider the vector differential equation

$$
\dot{x}=f(x, t), \quad x\left(t_{0}\right)=x_{0}
$$

where $x \in \mathbb{R}^{n}$ and $f: \mathbb{R}^{n} \times \mathbb{R} \rightarrow \mathbb{R}^{n}$ satisfies the following condition.

Condition $\mathbf{B}^{4)}: f$ is defined almost everywhere and is measurable in an open region $D \subset R^{n+1}$. Further, for every compact set $Q \subset D$, there exists an integrable $B(t)$ such that $\|f(x, t)\| \leq B(t)$ a.e. in $Q$.

Under Condition $B$, the local existence of the Filippov solution is assured ${ }^{4)}$.

Definition (Filippov solution ${ }^{4)}$ ). A vector function $x(t)$ is called a solution of (A. 1$)$ on $\left[t_{0}, t_{1}\right]$ if $x(t)$ is absolutely continuous on $\left[t_{0}, t_{1}\right]$, and for almost all $t \in\left[t_{0}, t_{1}\right]$

$$
\dot{x} \in K[f](x, t)
$$

where

$$
K[f](x, t)=\bigcap_{\delta>0} \bigcap_{\mu N=0} \overline{\operatorname{co}} f(B(x, \delta)-N, t) .
$$

$\bigcap_{\mu N=0}$ denotes the intersection over all sets $N$ of Lebesgue measure zero, $B(x, \delta)=\left\{y \in R^{n} \mid\|y-x\|<\delta\right\}$ and $\overline{\mathrm{co}}$ denotes the convex closure.

The uniqueness of the Filippov solution is one of fundamental problems as well as the existence of the solution. According to the reference ${ }^{4)}$, a uniqueness theorem for the equations with piecewise continuous right-hand side is provided.

Let a bounded region $G$ of the space $\mathbb{R}^{n}$ be divided by a smooth surface $S$ into the domains $G^{-}$and $G^{+}$. In $G$ for $T_{0}<t<T_{1}$ let the system of equation (A.1) be given, the right-hand side $f$ of which is measurable and bounded in the $(n+1)$-dimensional region $Q=G \times\left(T_{0}, T_{1}\right)$. For any constant $t \in\left(T_{0}, T_{1}\right)$ let the function $f$ be continuous in $x$ in $G^{-}$and $G^{+}$, and let there exist limiting values of the function $f$ under approach to an arbitrary point of the surface $S$ from the domain $G^{-}$and $G^{+}$. Suppose that for all $t$ in $G^{-}$and $G^{+}$

$$
\left\|\frac{\partial f}{\partial x}\right\| \leq K
$$

where $K$ does not depend on $t, x$. We will denote by $f^{-}$ and $f^{+}$the limiting values of the vector $f$ under approach to $S$ from $G^{-}$and $G^{+}$. Letters with the index $n$, for example $f_{n}^{+}$, will denote the projection (with the index) of the corresponding vector on the normal to the surface $S$ at the point $(t, x)$; the positive direction of the normal is always from $G^{-}$to $G^{+}$.

Theorem A.1 (Uniqueness of the solution). Let system (A.1) satisfy the above-mentioned conditions, and in addition let the surface $S$ be twofold smooth, let the function $f_{n}^{-}$and $f_{n}^{+}$be continuous in $t, x$ for $x \in S$, and let the vector $h=f^{+}-f^{-}$be continuously differentiable. If at least one of the inequalities $f_{n}^{-}>0$ or $f_{n}^{+}<0$ is satisfied at each point of $S$, not necessarily the same one at all points of the surface, then in the domain $G$ for the system (A. 1) we have right-hand uniqueness and continuous dependence of the solution on the initial conditions.

Under the concept of the Filippov solution, some related Lyapunov stability results have been proposed ${ }^{11)}$.

Theorem A.2 (Lyapunov ${ }^{11)}$ ). Let $\dot{x}=f(x, t)$ be essentially locally bounded and $0 \in K[f](0, t)$ in a region $\left\{x \in R^{n} \mid\|x\|<r\right\} \times\left\{t \mid t_{0} \leq t<\infty\right\} \subset D$. Assume that the existence and uniqueness of the Filippov solution are assured. Let $V: R^{n} \times R \rightarrow R$ be a regular function (See the reference $^{3)}$ for definition) satisfying $V(0, t)=0$ and $0 \leq V_{1}(\|x\|) \leq V(x, t) \leq V_{2}(\|x\|)$ in $D$ for some $V_{1}, V_{2} \in$ class $\mathcal{K}$. Now the set valued derivative $\dot{\tilde{V}}(x)$ is defined by

$$
\dot{\tilde{V}}(x(t), t)=\bigcap_{\xi \in \partial V(x(t), t)} \xi^{T}\left[\begin{array}{c}
K[f](x(t), t) \\
1
\end{array}\right] .
$$

where $\partial V(x, t)$ is the Clarke's generalized gradient ${ }^{3)}$ Then,

(1) $\dot{\tilde{V}}(x, t) \leq 0$ in $D$ implies $x(t) \equiv 0$ is a uniformly stable solution.

(2) If in addition, there exists a class $\mathcal{K}$ function $w(x)$ in $D$ with the property $\dot{\tilde{V}}(x, t) \leq-w(x) \leq 0$, then the solution $x(t) \equiv 0$ is uniformly asymptotically stable. Moreover, 
if $D=\mathbb{R}^{n}$ and $V_{1}$ is radially unbounded, then $x=0$ is

globally uniformly asymptotically stable.

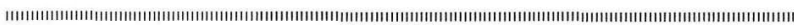

Takashi Naкакuкi (Student Member)

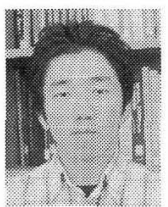

He received the B.E. and M.S. degrees from Sophia University, Japan, in 1997 and 1999, respectively. From 1999, he worked for Sony corporation to develop algorithms of image processing for four years. He is currently a doctoral course student of Graduate school of Science and Technology, Sophia University. His research interests include nonlinear systems, robust control theory, intelligent control and image processing.

\section{Katsutoshi Tamura (Member)}

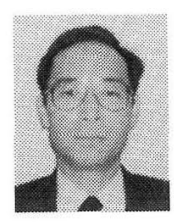

After receiving Master Degree from Nagoya University, he was appointed to a research associate there, and began his $\mathrm{Ph}$. D research on optimal control theory and algorithm to obtain optimal control input to linear and nonlinear systems. After this he moved to Sophia University as a lecturer and got promotion to an associate professor and to a professor. Now, he is working on adaptive control and nonlinear control.

Tielong Shen (Member)

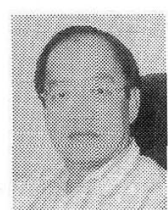

Tielong Shen received the B.E. and M.S. degrees in Automatic Control from Northeast Heavy Machinery Institute, China in 1982 and 1986, respectively, and the $\mathrm{Ph}$. D. degree in Mechanical Engineering from Sophia University, Tokyo, Japan, 1992. From March 1986 to March 1989, he served as a teaching assistant and then a lecture in the Department of Automatic Control, Northeast Heavy Machinery Institute. Since April 1992, he was been a faculty member as an Assistant Professor of Department of Mechanical Engineering at Sophia University. He is the author/coauthor of two textbooks. His current research interests include $H_{\infty}$ control theory, robust control of linear and nonlinear systems and its applications in mechanical systems. 